\title{
Combination Method of Stochastic Load Processes
}

\author{
XIAO-HONG WANG \\ Zhejiang Guangchuan Engineering Consulting Co., Ltd, Hangzhou, 310020, China \\ 329013096@qq.com
}

Keywords: load combination; limit state design; cumulative distribution function.

\begin{abstract}
When more than one loads act on a structure, the combination of stochastic loads is generally considered in the design of the structure, Based on the theoretical derivation and numerical calculation analysis to the process of two common loads in Poisson square wave and Poisson impulse this paper presents the combination method of loads i.e. The maximum intensity function of load combination is expressed by the cumulative distribution function in the limit state design. In addition, the result calculated during the limit state design is compared with those calculated by Turkstra and Monte Carlo methods and the analytical results show that the method proposed in this paper is feasible in the calculation of load combination.
\end{abstract}

\section{Introduction}

In addition to the dead load, engineering structures are usually under a variety of variable load. The combination of the load must be considered. Because of the emergence of various kinds of variable load is in accordance with the time, space, and change of stochastic process, so its maximum value can't appear at the same time. From the perspective of mathematical statistics, after the superposition of load combination problems involving multiple random variables statistical characteristics of the problem.

\section{The load random process model}

Engineering of the common load random process is divided into two categories, which are poisson process of rectangular wave and pulse poisson process. For poisson process of rectangular wave, within the duration of the load amplitude remains unchanged, and the change point of the process can be described by poisson process. That is to say, before t the times of load is the poisson distribution.

$$
P[N(t)=k]=\frac{(\lambda t)^{k}}{k !} e^{-\lambda t}
$$

In which, the parameter $\lambda$ is the average of the load change the number of times per unit time.

Poisson process of rectangular wave loads during the process of each pulse duration is subject to exponential distribution of random variables. Usually adopt the persistence live load poisson process of rectangular wave as a stochastic process model. If only for changes in load time, the 
process available pulse poisson process model (Fig. 2). In the limit state design, wind load and earthquake load are available poisson model describes pulse process.

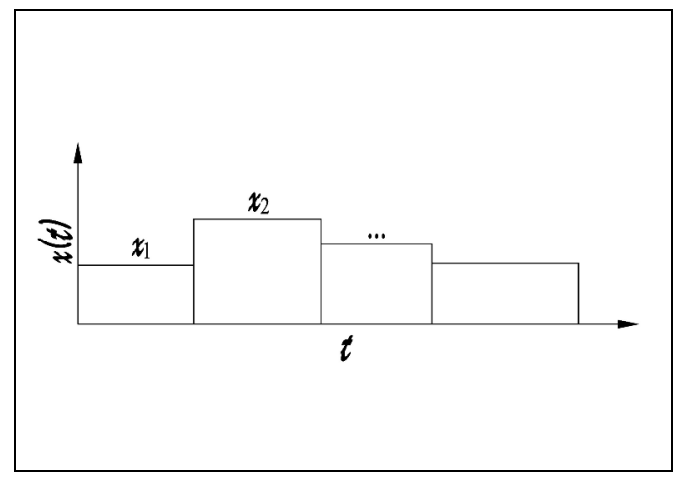

Fig. 1 Poisson process of rectangular wave model

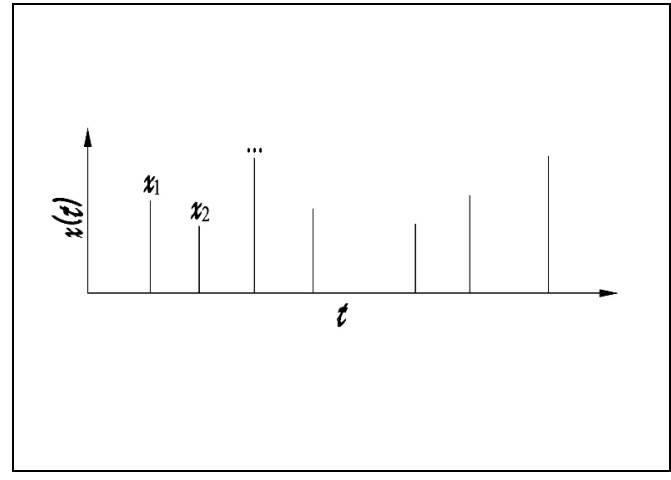

Fig. 2 Poisson impulse process model

\section{The method of load combination}

In this paper, the different load combination method was proposed. Let $X_{1}(t)$ be the poisson process of rectangular wave, $X_{2}(t)$ be The poisson process of pulse. The combined load can be expressed as

$Z(t)=X_{1}(t)+X_{2}(t)$

The probability distribution of the maximum of two load combination can be expressed as

$$
F_{Z_{\max j}}(z)=\int_{0}^{\infty} F_{X_{2 \text { MAXJ }}}\left(z-x_{1}\right) f x_{1}\left(x_{1}\right) d x_{1}
$$

Where, $f_{X 1}(x 1)$ represent the probability distribution function of the Poisson rectangular wave process. One moment (average) approximation can be shown as follows

$$
\begin{aligned}
& F_{Z_{\max } \mid N_{x_{1}}}\left(z \mid n_{x_{1}}\right) \approx \\
& \prod_{j=1}^{n_{x_{1}}+1} \int_{0}^{\infty} \exp \left\{-\lambda_{x_{2}} \frac{t_{L}}{n_{x_{1}}+1}\left[1-F_{x_{2}}\left(z-x_{1}\right)\right]\right\} \times f_{x_{1}}\left(x_{1}\right) d x_{1} \approx \\
& {\left[\int_{0}^{\infty} \exp \left\{-\frac{\lambda_{x_{2}} t_{L}}{n_{x_{1}}+1}\left[1-F_{x_{2}}\left(z-x_{1}\right)\right]\right\} \times f_{x_{1}}\left(x_{1}\right) d x_{1}\right]^{n_{x_{1}}+1}}
\end{aligned}
$$

Let $n_{x 1}$ be a random variable, the mean can be shown as follows

$$
E\left(n_{x 1}\right)=\lambda_{x 1} t_{L}
$$

The formula (5) generation into the formula (4), formula (6) can be shown as follows 


$$
\begin{aligned}
& F z_{\max }(z)=E\left[F z_{\max \mid N x_{1}}\left(z \mid n_{x_{1}}\right)\right] \\
& \approx\left[\int_{0}^{\infty} \exp \left\{-\frac{\lambda x_{2} t_{L}}{E\left(n_{x_{1}}\right)+1}\left[1-F x_{2} \times\left(z-x_{1}\right)\right]\right\} f x_{1}\left(x_{1}\right) d x_{1}\right]^{E\left(n_{x_{1}}\right)+1} \\
& \approx\left[\int_{0}^{\infty} \exp \left\{-\frac{\lambda x_{2} t_{L}}{\lambda x_{1} t_{L}+1}\left[1-F x_{2} \times\left(z-x_{1}\right)\right]\right\} f x_{1}\left(x_{1}\right) d x_{1}\right]^{\lambda x_{1} t_{L}+1}
\end{aligned}
$$

\section{Case Study}

Live load $X_{1}(t)$ is a poisson process of rectangular wave, the average annual rate of change: 0.125 , load magnitude obey the extremum distribution, the mean:4.0 coefficient of variation: 0.4 . Wind load $X_{2}(t)$ is a poisson process of pulse, load magnitude obey the extreme value distribution, average :2.0, the same Different coefficient :0.4;Structural design reference period $\mathrm{t}: 50 \mathrm{a}$, the biggest distribution combined load can be shown as follows

$F_{\text {max }}(z)=X_{1}(t)+X_{2}(t)$.

The calculated results draw as follows (Fig. 3)

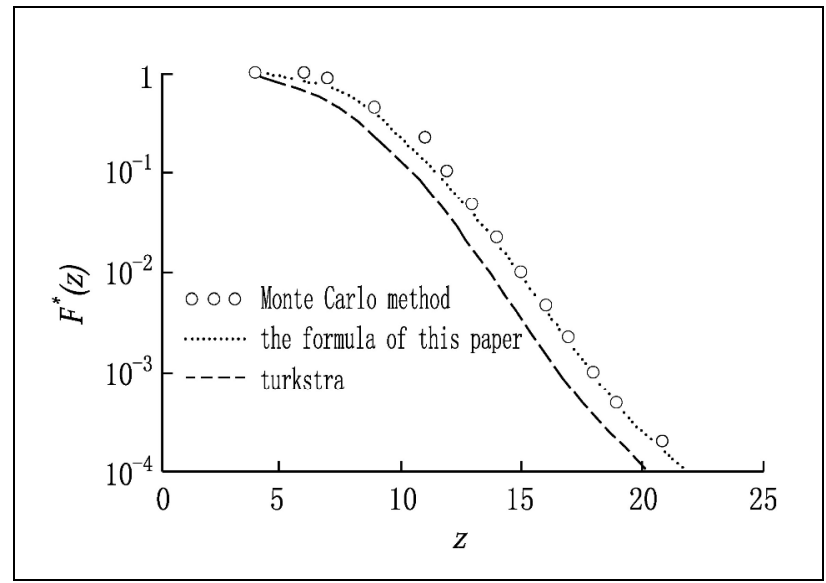

Fig. 3 The comparison of calculation results

By Fig. 3, the paper puts forward the formula to calculate the probability of the value and monte carlo method simulation results are in good agreement.

\section{Conclusions}

Through the examples, this article put forward the method of the calculation results and calculation results Turkstra method and monte carlo method has carried on the comparative analysis, to verify the proposed random process model of engineering load combination method is feasible.

\section{References}

[1]Pearce H T, Wen Y K.Stochastic combination of load effects.J Str Div,1984,110(7), p. 1613-1629 
[2]Wen Y K.Statistical combination of extreme loads.J Str Div.1977,103(5), p. 1079-1093

[3]Melchers R E.Structural reliability analysis and prediction.2nd West Sussex(UK):John Wiley and Sons, 1999

[4]Sakamoto J,Mori Y,Sekioka T.Probability analysis method Using Fast Fourier transform and its application.Structural Safety.1997,19, p. 21-36 